Mingbao PANG, Ph.D. ${ }^{1}$

(Corresponding author)

E-mail: pmbpgy@hebut.edu.cn

Xing WANG, Master student ${ }^{1}$

E-mail: wxx951027@163.com

Lixia MA, Senior engineer ${ }^{2}$

E-mail: 344124531@qq.com

${ }^{1}$ School of Civil and Transportation

Hebei University of Technology

Tianjin, 300401, China

2 Planning and Design Institute of Handan City

Handan, 056001, China
Transport Engineering Original Scientific Paper Submitted: 23 Nov. 2020 Accepted: 12 Mar. 2021

\title{
TRANSIT ROUTE PLANNING FOR MEGACITIES BASED ON DEMAND DENSITY OF COMPLEX NETWORKS
}

\begin{abstract}
The aim of this work is to investigate the simplification of public transport networks (PTNs) for megacities and the optimisation of route planning based on the demand density of complex networks. A node deletion rule for network centre areas and a node merging rule for network border areas in the PTN are designed using the demand density of complex networks. A transit route planning (TRP) model is established, which considers the demands of direct passengers, transfer passengers at the same stop and transfer passengers at different stops, and aims at maximising the transit demand density of a PTN. An optimisation process for TRP is developed based on the ant colony optimisation (ACO). The proposed method was validated through a sample application in Handan City in China. The results indicate that urban PTNs can be simplified while retaining their local attributes to a great extent. The hierarchical structure of the network is more obvious, and the layer-by-layer planning of routes can be effectively used in TRP. Moreover, the operating efficiency and service level of urban PTNs can be enhanced effectively.
\end{abstract}

\section{KEYWORDS}

urban public transport; network simplification; transit route planning; demand density of complex network; ant colony optimisation.

\section{INTRODUCTION}

The planning level of public transport networks (PTNs) will directly affect the operating efficiency and service level of the network [1]. As the main part of PTN planning, transit route planning (TRP) has been extensively studied [2], where the meth- od of "one-by-one wiring, optimised for network" method adopted by Zhou K. et al. in [3] has been widely used because of its simple operation. For megacity PTNs, the "layer-by-layer, route-byroute" method proposed by Carrese et al. in [4] has been widely used. However, the overall planning of a PTN is highly inefficient because of the complexity and time-consuming nature of the route searching phase, especially for megacities with huge public transport systems. To enhance the efficiency, many heuristic algorithms have been developed, e.g. the alternating objective genetic algorithm [5], tabu search heuristic algorithm [6], and improved ant colony optimisation (ACO) algorithm [7]. However, with the maturity of the algorithm, it becomes difficult to continuously improve planning efficiency, especially megacity PTN planning. It is generally known that the complexity of a path search algorithm is directly proportional to the number of nodes in the network [8]. Therefore, it is feasible to reduce the number of nodes and edges to simplify the network (reduce network size) by studying the network topology.

Complex networks are widely used to describe and study a variety of systems in the real world, including the Internet, neural networks, traffic, and transportation [9]. For megacity public transport systems, network complexity analysis and its evolution, e.g. robustness and cascading failure characteristics, have been widely studied [10]. Moreover, some detour strategies have been studied aiming to avoid congestion nodes in the network reasonably in the route planning process [11]. Several studies 
Pang M, Wang X, Ma L. Transit Route Planning for Megacities Based on Demand Density of Complex Networks

have shown that these methods are feasible for improving network operation efficiency. Based on the missing link prediction and the spurious existing link identification, Zhang et al. proposed a framework of the auxiliary optimisation method for the transit network [12]. Jia et al. developed a model based on betweenness to optimise the transportation network [13]; however, this has not been further studied due to complex network theory.

Simplifying the complex network is an efficient method for understanding, analysing, and visualising networks of large real-world systems [14]. Several authors have proposed a broad collection of simplification methods that can be classified into two general types: sampling methods [15] and merging methods [16]. Blagus et al. tested different simplification methods on 30 real-world networks, and found that the sampling method has low computational complexity and strong adjustability, but it also causes damage to network connectivity, whereas the merging method does the opposite [17]. The cluster-growing renormalisation (CG) method employed by Callos et al. in [18] and the balanced propagation (BP) method used by Subelj et al. in [19] have both been proved well in reducing the network size while maximising the network self-similarity [20]. In previous studies, the simplification methods for megacity PTNs were limited. The main idea of the established methods is to delete the node pairs whose node spacing is less than the specified value (i.e., $0.3 \mathrm{~km}$ ) [20]. Little attention has been paid to the transit demand between nodes. If the TRP is carried out on the simplified PTN, all the actual transit demand will not be satisfied in the final planned network. Therefore, a simplified method based on the transit demand density on the edge of the network [21] is employed to simplify the PTN.

The distribution of stops in the PTN of a megacity has the characteristics of dense centres and sparse borders [22]. In network centre areas, the service scope of two bus stops often overlaps [23]. The bus stops in network border areas are very important for residents to travel, which cannot be ignored in the process of TRP [24]. Therefore, there are two ways to simplify the PTN. The first is to simplify network border areas by merging nodes into super-nodes, where the connection relationship between merged nodes is relatively fixed. The second is to delete nodes based on the node deletion rule to simplify the network centre areas. The node deletion rule is aimed at the node pairs whose bus stops spacing is less than the passenger acceptable walking distance, i.e., $0.3 \mathrm{~km}$, and after one node of a pair is deleted, the retained node undertakes its passenger transit demand.

For a megacity with a large population, the key to TRP is meeting the travel demands of different passengers and optimising the PTN structure [25]. Therefore, the TRP process is divided into three stages according to the features of transit routes [21]. It aims to maximise the number of travellers per unit length, i.e., the transit demand density, according to the route length and the number of travellers on the route [26]. The skeleton routes are first planned according to the transit trip origin-destination (OD). Then, the trunk routes are planned according to the transit trip OD left by the planned skeleton routes. Lastly, the transit trip OD left by the above planned routes will be covered by planning the coverage routes as possible. In TRP, this is difficult to achieve through classical optimisation techniques [27]. Both the TRP process and the ant foraging process are the probability selection problem [28], hence, an optimisation solution process based on ACO is proposed.

Due to the above analysis, a simplification method for a megacity PTN is proposed, which considers both the transit demand and the transit distance between nodes. The network centre areas are simplified by deleting nodes. The network border areas are simplified by merging nodes into super-nodes, and the location relationship between super-node and other nodes is defined. On the simplified network, TRP will be carried out with the goal of maximising the total transit demand density on the route. The total transit demand density on the route is calculated by dividing the total transit demand by the route length, where the total transit demand includes the direct trips, the transfers at the same stop, and especially the transfers at the different stops. Based on the ACO, the transit routes will be planned in the order of skeleton routes, trunk routes, and coverage routes. The proposed method will be verified through simulation experiments.

The remainder of this paper is organised as follows. In Section 2, the simplification rules of the PTN are presented. Section 3 establishes the TRP model and Section 4 provides a concrete optimisation algorithm based on the ACO. An application is provided in Section 5. Finally, the conclusions of the study are summarised. 


\section{SIMPLIFICATION OF PTN}

A megacity PTN was used as the researched object (Figure 1), the set of network nodes $N$ consists of the existing bus stops, $l_{i j}$ represents the road distance between two stops. If $l_{i j}<0.3 \mathrm{~km}$, the node pair is connected by a dashed line. If $0.3 \mathrm{~km}<l_{i j}<0.8 \mathrm{~km}$, the node pair is connected by a solid line [21]. $E_{1}$ and $E_{2}$ represent the set of dashed and solid edges, respectively. Figure 1 is a sample network, in which $1,2,7$, and 8 are the terminal nodes (bus stations), and 3, 4, 5, and 6 are intermediate nodes (bus stops).

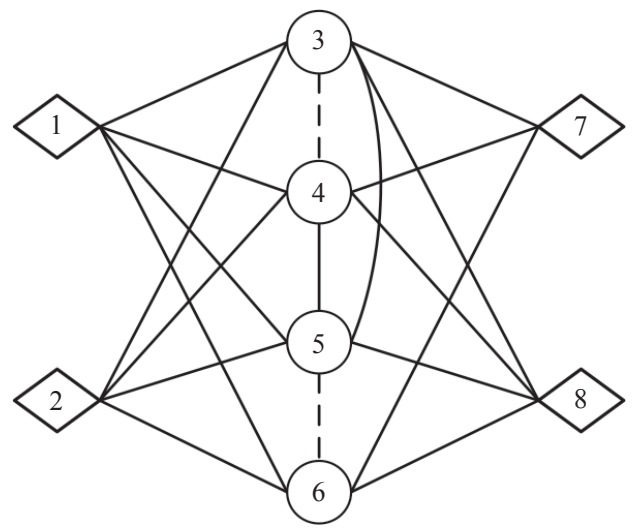

Figure 1-An example network

\subsection{Simplification rule of network centre areas}

For the node pair linked by dashed lines in the network centre areas, a node deletion rule is instituted and described by Figure 2 .

The set of nodes connected to nodes $i$ and $j$ by solid lines are $\Omega_{i}$ and $\Omega_{j}$, respectively, where the node $k$ is in $\Omega_{i}$ and the node $h$ is in $\Omega_{j}$. If $\exists l_{k j}>0.8 \mathrm{~km}$ and $\exists l_{i h}>0.8 \mathrm{~km}$ (Figure $2 a$ ), then neither node is deleted. If $\forall l_{k j}<0.8 \mathrm{~km}$ and $\exists l_{i h}>0.8 \mathrm{~km}$ (Figure 2b), then deleting node $i$ and its passenger transmission function is replaced by node $j$. Similarly, if $\exists l_{k j}>0.8 \mathrm{~km}$ and $\forall l_{i h}<0.8$

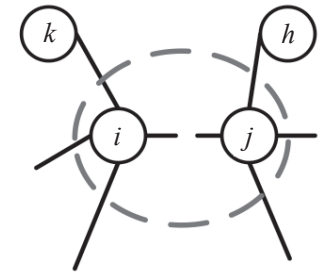

a)

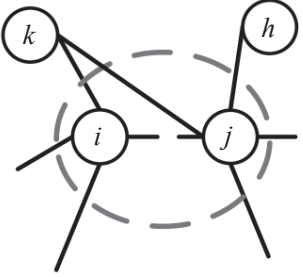

b)

$\mathrm{km}$ (Figure 2c), then deleting node $j$ and its passenger transmission function is replaced by node $i$. If $\forall l_{k j}<0.8 \mathrm{~km}$ and $\forall l_{i h}<0.8 \mathrm{~km}$ (Figure $2 d$ ), it is necessary to calculate the average increase in transit demand density on the solid edges around another node when a node is deleted, and this can be calculated by Equation 1.

$$
\begin{aligned}
& \eta_{i}=\sum_{k \in \Omega_{i}} \frac{P_{i j}+k_{i} P_{i k}}{k_{i}^{2} \cdot L_{j k}} \\
& L_{m n}=l_{m n}\left[1+x_{m n} \cdot a\left(\frac{Q_{m n}}{C_{m n}}\right)^{b}\right]
\end{aligned}
$$

where $P_{i j}$ (the unit is person) denotes the number of passengers between stops $i$ and $j . k_{i}$ is the node degree of $i . L_{m n}$ (the unit is kilometer) represents the delay distance. $l_{m n}, Q_{m n}$, and $C_{m n}$ represent the actual distance (the unit is kilometer), traffic volume (the unit is person), and capacity (the unit is person) of the road section $m n$, respectively. $x_{m n}$ is a binary variable that represents whether there are bus lanes on $m n$, if so, it is set to 0 , otherwise it is set to $1 . a$ and $b$ are adjustment parameters.

If $\eta_{i}>\eta_{j}$, then the node $i$ is deleted, otherwise, the node $j$ is deleted. If $\eta_{i}=\eta_{j}$, then any node can be deleted. Meanwhile, the O-D matrix must be updated after deleting a node. Take deleting the node $i$ in Figure $2 d$ as an example to explain the updating process of the O-D matrix. First, the $P_{i j}$ is divided into $k_{j}$ equal portions and added to $P_{j k}$. Then, the row and column corresponding to node $i$ are added to those of node $j$, respectively, and the row and column of node $i$ are deleted to obtain the updated O-D matrix.

\subsection{Merging rule of network border areas}

Owing to the sparse distribution of nodes at the network border area and that the order of connections between multiple nodes is relatively fixed, merging these nodes into super-nodes is feasible. The nodes contained in the super-node are denoted as set $\omega$. As shown in Figure 3, the super nodes $k_{1}$ and

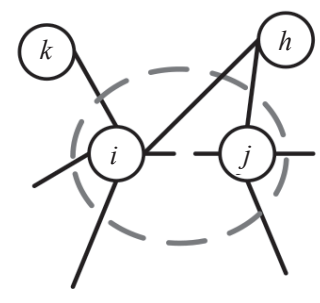

c)

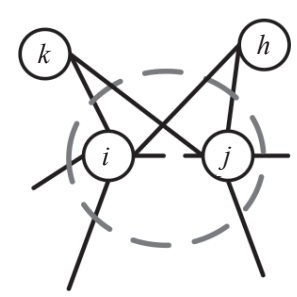

d)

Figure 2-An example of network centre area node deletion rule 

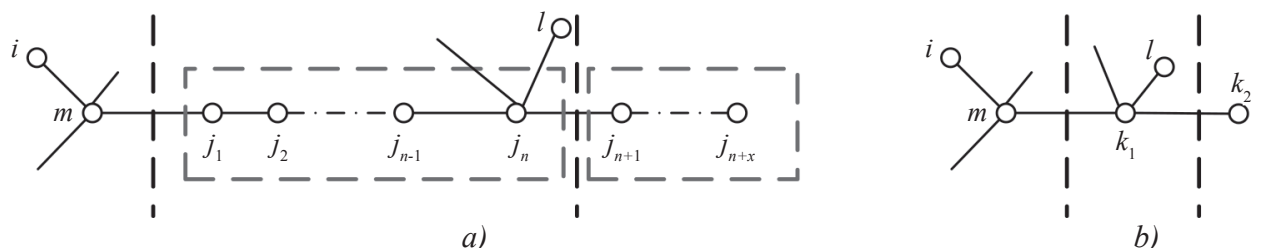

Figure 3-An example of network border area simplification

$k_{2}$ in Figure $3 b$ are generated by merging the nodes in Figure $3 a$, and the node sets $\omega_{1}=\left\{j_{1} j_{2}, \ldots, j_{n-1} j_{n}\right\}$ and $\omega_{2}=\left\{j_{n+1} j_{n+2}, \ldots, j_{n+x}\right\}$ are generated.

To reduce the impact of merging nodes on the overall network structure, the location identification of the super-node is defined. Such as in Figure $3 b$, the location of super-nodes $k_{1}$ and $k_{2}$ corresponds to the location of nodes $j_{n}$ and $j_{n+x}$ in Figure $3 a$, respectively. The transit demand between nodes and super nodes in the network can be calculated using Equation 3. The transit demand between super nodes can be calculated by Equation 4. When updating the O-D matrix, delete the rows and columns of nodes that have been merged, and add the rows and columns of the super nodes to the matrix.

$$
\begin{aligned}
& P_{i k_{1}}=\sum_{m=1}^{n} P_{i j_{m}} \\
& P_{k_{1} k_{2}}=\sum_{m=1}^{n} \sum_{q=1}^{x} P_{j_{m j_{n}+q}}
\end{aligned}
$$

\section{TRP MODEL}

The TRP model proposed in this study was developed to maximise the total transit demand density of the route, which is the number of transit demands divided by the distance. Total transit demand includes direct passengers, transfer passengers at the same stop and transfer passengers at different stops, which can be calculated by Equations 5, 6, and 7, respectively. The transit distance is the delay length of the route, and assumes that the road section is equally divided between stops. In the planning process, $R_{o d}$ denotes the route being constructed, and $R_{m n}$ denotes the route in the optimised transit network.

$$
P_{R o d}^{\mathrm{direct}}=\sum_{i \in N} \sum_{j \in N} P_{i j} \mathrm{R}_{i j}^{R_{j} d}
$$

$P_{R_{o d}}^{\mathrm{transer,s}}=\sum_{i \in N} \sum_{j \in N} P_{i j} z_{i j}^{R o d, R_{m n}} \delta_{i j}^{\mathrm{transfer}, \mathrm{s}}$

$$
P_{\text {Rod }}^{\text {transer, }}=
$$

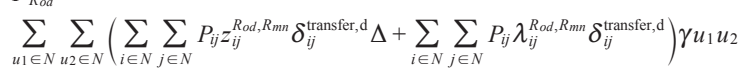

$\delta_{i j}^{\text {transfer, }{ }^{*}}= \begin{cases}1 & \left(l_{i j}^{\text {transfer, }{ }^{*}} / D i s_{i j}\right)<3 \\ 0 & \text { otherwise }\end{cases}$

$$
l_{i j}^{\mathrm{transer}, \mathrm{s}}=\min _{u=\pi_{R_{o d}} \cap \pi_{R_{m n}}}\left(l_{i u}^{R o d}+l_{l j}^{R_{m i}}\right)
$$

$l_{i j}^{\text {transfer, }}=\min _{u \in \pi_{R_{0 o d}, u 2} \in \pi_{R_{m n}}}\left(l_{i u 1}^{R o d}+l_{u 1 u_{2}}+l_{u 2 j}^{R m n}\right)$

where $P_{R_{o d}}^{\mathrm{direc}}, P_{R_{o d}}^{\mathrm{transer}, \mathrm{s}}$, and $P_{R_{o d}}^{\mathrm{transer}, \mathrm{d}}$, (the unit is person) denote the number of direct trips, the number of transfer passengers at the same stop, and the number of transfer passengers at different stops, respectively. $y_{i j}^{R o d}$ indicates whether the stops $i$ and $j$ are on route $R_{o d}$, if so, it is set to 1 , otherwise, it is set to $0 . z_{i j}^{R_{o d}, R_{m n}}$ represents whether the trip from stop $i$ to stop $j$ (assume that stops $i$ and $j$ are on the routes $R_{o d}$ and $R_{m n}$, respectively) requires transfer, if so, the value is 1 , otherwise, it is 0 . Figure $4 a$ shows the process of passengers transfer at the same stop. On the right side of Equation 7, the first and second parts in brackets describe the transfer process shown in Figures $4 b$ and $c$, respectively. $\Delta$ indicates whether the travel distance that passengers transfer at different stops is shorter than that transfer at same stop, if so, it is set to 1 , otherwise, it is set to $0 . \lambda_{i j}^{R o d, R m n}$ denotes whether there are intersections between the routes $R_{o d}$ and $R_{m n}$, if so, it is set to 0 , otherwise, it is set to 1. $\gamma_{u_{1} u_{2}}$ denotes whether the distance between

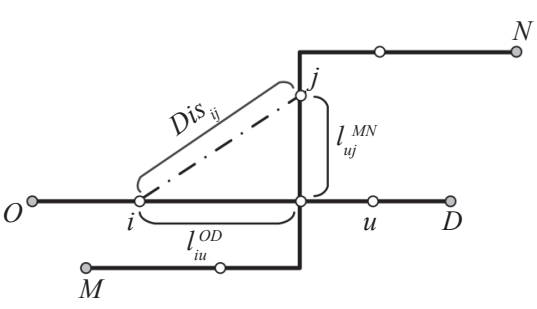

a)

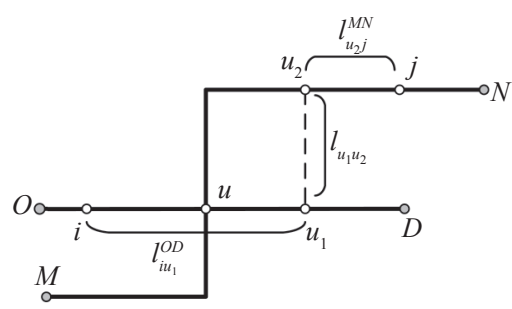

b)

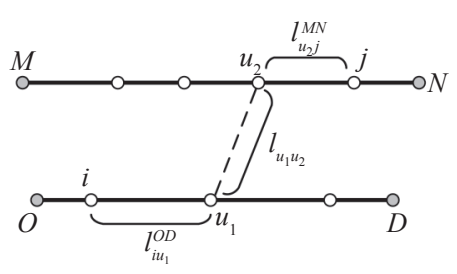

c)

Figure 4-Examples of transfer at the same and different stops 
the stops $u_{1}$ and $u_{2}$ (assume that stops $u_{1}$ and $u_{2}$ are on the routes $R_{o d}$ and $R_{m n}$, respectively) is within the acceptable walking distance of passengers, if so, the value is 1 , otherwise, it is $0 . \delta_{i j}^{\text {transfer, }{ }^{*}}$ indicates whether the distance between two stops is too far, if so, it is set to 0 , otherwise, it is set to $1 . D i s_{i j}$ (the unit is kilometer) denotes the shortest distance between stops $i$ and $j$. The upper limit of $l_{i j}^{\text {transer, }}$ * $/$ Dis $i j$ is determined by the actual situation of China, and it is set to 3. $\pi_{R_{o d}}$ and $\pi_{R_{m n}}$ denote the node set that the route $R_{o d}$ and $R_{m n}$ pass through, respectively.

According to different constraints, TRP can be divided into three stages: skeleton routes, trunk routes, and coverage routes planning. To maximise the total transit demand density of the route, the proposed model can be formulated as follows:

$$
\begin{aligned}
& \operatorname{Max} \sum_{R o d} D_{R_{o d}} \\
& D_{\text {Rod }}=\frac{P_{R_{o d}}^{\mathrm{direct}}+w_{1} P_{R_{o d}}^{\mathrm{tranfer}, \mathrm{s}}+w_{2} P_{R_{o d}}^{\mathrm{trans}, \mathrm{d}}}{L_{R_{o d}}}
\end{aligned}
$$

$$
\begin{aligned}
& \text { s.t. } \\
& o, d \in s \\
& 5 \mathrm{~km}<l_{R_{o d}}<15 \mathrm{~km} \\
& Q_{R_{o d}}=P_{R_{o d}}^{\text {direct }}+P_{R o d}^{\text {transfer, }}+P_{R_{o d}}^{\text {transfer, }}>Q_{\text {min }} \\
& \text { for bus skeleton line design }\left\{\begin{array}{l}
w_{1} \neq 0, w_{2}=0 \\
l_{\text {od }} / D i s_{\text {od }}<1.2
\end{array}\right. \\
& \text { for bus trunk line design }\left\{\begin{array}{l}
w_{1} \neq 0, w_{2}=0 \\
l_{R_{o d}} / D i s_{R_{o d}}<1.5
\end{array}\right. \\
& \text { for bus coverage line design }\left\{\begin{array}{l}
w_{1} \neq 0, w_{2} \neq 0 \\
l_{R_{o d}} / D i s_{R_{o d}}<1.5
\end{array}\right.
\end{aligned}
$$

where $R$ is a set of all possible routes. $D_{R_{o d}}($ the unit is person $/ \mathrm{km}$ ) denotes the total transit demand density on route $R_{o d}$. $w_{1}$ and $w_{2}$ are used to reflect the importance of transfers at the same and different stops in terms of total transit demands, respectively. $s$ is the set of origins and destinations. $Q_{R_{o d}}$ (the unit is person) represents the total transit demand, and $Q_{\min }$ (the unit is person) is the minimum total transit demand on the route.

\section{OPTIMISATION ALGORITHM BASED ON ACO}

The specific steps of the algorithm are as follows:

\section{Step 1. Initialisation}

At the beginning of TRP, it is necessary to initialise the network and the pheromone matrix. For a simplified network, it is necessary to identify the terminal nodes and intermediate nodes, ensure that there are no routes in the network. In the initial network, it is necessary to set an initial weight to each edge, that is, to initialise the pheromone matrix, and the value of each element $\tau_{0}$ in the matrix is the same constant that is not equal to 0 .

\section{Step 2. Choosing the OD pair}

Before searching the route, a feasible OD pair must be selected. If all routes between an OD pair cannot meet the constraints, then the OD pair will not be considered.

\section{Step 3. Search route}

In the process of searching routes, the ant chooses the next node by taking account into both the pheromone intensity $\tau_{i j}$ and the visibility value $F_{i j}$ of the corresponding links. The intensity of pheromone is related to the planning objective [27]. The visibility value is calculated by gravity. The probability that the $k$ th ant on node $i$ moves to the node $j$ is given by Equation 13 .

$\theta_{i j}(k)= \begin{cases}\frac{\tau_{i j}^{\alpha} F_{i j}^{\beta}}{\sum_{h \notin t a b u_{i}} \tau_{i h}^{\alpha} F_{i h}^{\beta}} & \text { if } j \notin t a b u i, \\ 0 & \text { otherwise }\end{cases}$

$F_{i j}=\frac{c_{i} \cdot c_{j}}{l_{i j}^{2}}$

$c_{i}=\sum_{k \in \Omega_{i}} \frac{P_{i k}}{L_{i k}}$

where $c_{i}$ denotes the node weight. $\alpha$ and $\beta$ are parameters. $t a b u_{i}$ is the set of nodes that the $k$ th ant on node $i$ cannot access, including the nodes that have been accessed by the $k$ th ant and the nodes that cannot satisfy the distance constraint.

\section{Step 4. Updating the pheromone}

When determining a route, it is necessary to update the network pheromone at the same time, and the updating process of the network pheromone can be divided into two parts. The first part simulates the reduction of pheromone due to natural volatilisation. The second part is the pheromone increments of the edge due to ants passing by, which is related to the optimisation objective. The pheromone updating equation is as follows:

$\tau_{i j}^{\prime}=(1-\rho) \cdot \tau_{i j}+\sum_{k=1}^{M} \Delta \tau_{i j}^{k}$

where $\tau_{i j}$ denotes the existing pheromones intensity in edge $(i, j) \cdot \tau_{i j}^{\prime}$ denotes the intensity of the pheromone in edge $(i, j)$ after updating. $\rho$ is a constant, representing the speed of volatilisation. $\Delta \tau_{i j}^{k}$ denotes the pheromone increment on the edge $(i, j)$ of the 
route that searched by the $k$ th ant. $M$ is the total number of ants. The ant-cycle method is selected to calculate the pheromone increment as follows [28]:

$\Delta \tau_{i j}^{k}= \begin{cases}\frac{D_{R_{o d}}^{k}}{\mu} & \text { section }(i, j) \text { on the route } R_{o d} \\ 0 & \text { otherwise }\end{cases}$

where $\mu$ is a constant. $D_{R o d}^{k}$ denotes the objective function value of the route discovered by the $k$ th ant.

\section{Step 5. Generating the set of alternative routes}

When all ants in the network have finished the route searching, the route with maximum transit demand density between the OD pair is determined as the alternative route. The route searching between the current OD pair is end, and return to Step 2 selects the next feasible OD pair. Repeat Steps 2-5 until all alternative routes are found. The set of the alternative routes is expressed as $S$.

\section{Step 6. Selecting and adding route to the network}

Select the route with maximum transit demand density in the set $S$, add it to the network, and delete the others.

\section{Step 7. Transit passenger assignment}

In the process of TRP, when a new route is added to the network, it is necessary to assign the passenger demand at the same time. Assuming that there are $N$ routes in the existing network, when adding the $(N+1)$ th optimal route to the network the specific steps of the assignment are as follows [21].

Step 7.1. Add the $(N+1)$ th optimal route to the network and calculate the departure frequency (the departure frequency value is fixed and is different on different levels of routes).

Step 7.2. Divide the initial O-D matrix into $X$ equal parts, where the larger the value of $X$ is, the closer the assignment result is to the equilibrium assignment method. However, if the value of $X$ is too large, the distribution efficiency will be seriously affected. Step 7.3. Assign one part of the O-D matrix to the route.

Step 7.3.1. Select any OD pair, and assign the passenger demand to the minimum hyper-path between the OD pair. The hyper-path is determined by calculating the travel time of the path, which includes the running time of the bus and the waiting time of the passengers at stops (the waiting time $=1 / 2 \cdot 60$ /frequency).

Step 7.3.2. Recalculate the travel time of each route in the network. Check whether the total transit demand of each section on the route satisfies the capacity limit, if so, turn to Step 7.4. Otherwise, an infinite waiting time is set for the section that has reached or exceeded the capacity limit to ensure that it is no longer selected for the future assignment.

Step 7.3.3. Repeat Steps 7.3.1-7.3.2, until the current part is assigned to the network.

Step 7.4. Repeat Step 7.3 until assigning the entire O-D matrix.

Step 7.5. Update the frequencies of the $N+1$ routes.

\section{Step 8. Conditions for ending the search}

If there is no route that meets the constraints, stop and go to Step 10. Otherwise return to Step 2.

\section{Step 9. Node resetting}

Expand the super nodes in the optimal network and reset the merged nodes to their positions before merging.

\section{APPLICATION ANALYSIS}

The public transport system data of the city of Handan in China, with a population of 2.2 million, was used for the test. The data (from Planning and Design Institute of Handan City) includes 96 bus lines, 97 bus stations, and 1006 bus stops. When establishing the PTN, the stops with the same name but different geographical positions are different nodes. The PTN contains 1108 nodes, 3169 solid edges, and 544 dashed edges. In the experiment, when planning the skeleton routes, trunk routes, and coverage routes, the values of $Q_{\min }$ are 6000, 4000, and 2000, respectively, and other parameter value settings are listed in Table 1.

Table 1 - The value of parameter

\begin{tabular}{|c|c|}
\hline \hline Parameter & Value \\
\hline \hline$a$ & 0.68 \\
\hline$b$ & 2.48 \\
\hline$\tau_{0}$ & 1 \\
\hline$\alpha$ & 2 \\
\hline$\beta$ & 1 \\
\hline$\rho$ & 0.1 \\
\hline$M$ & 300 \\
\hline$\mu$ & 100 \\
\hline$X$ & 50 \\
\hline
\end{tabular}

\subsection{Simplifying the PTN}

After simplification, 17 nodes were deleted, and 179 nodes were merged to form 57 super-nodes; the network contains 912 nodes, 2911 


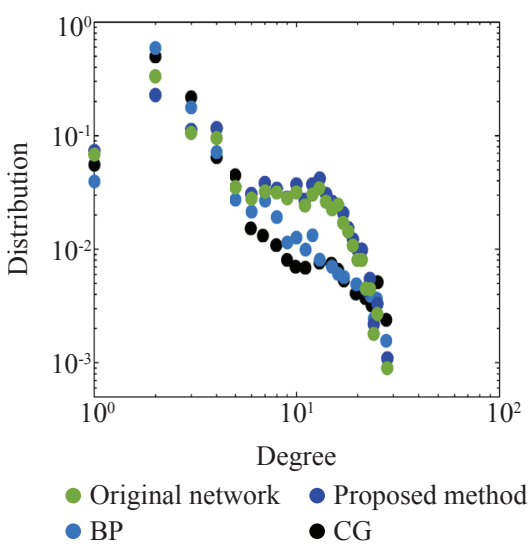

a)

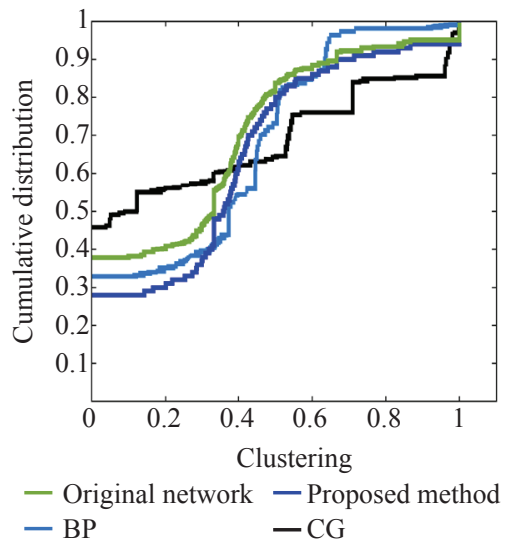

b)

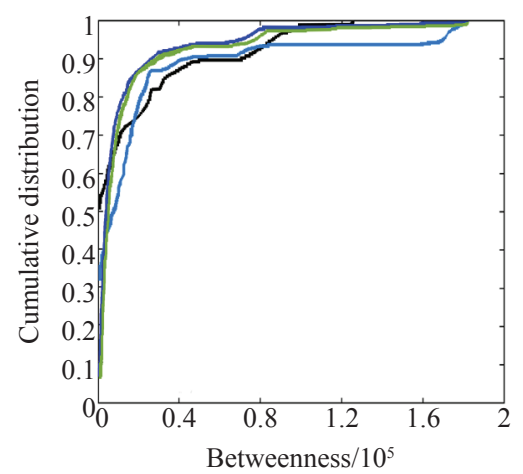

- Original network - Proposed method $\begin{array}{ll}-\mathrm{BP} & -\mathrm{CG}\end{array}$

c)

Figure 5 -Comparison of the local properties for the original and simplified network

solid edges, and 527 dashed edges. In the original network, there are at least two dashed lines around the deleted nodes, and the node degree is less than three. These nodes are all non-hub nodes in the network. Through the OD matrix, it is found that the bus stops corresponding to the deleted nodes have little transit demand in the PTN. After deleting the nodes, the $300 \mathrm{~m}$ and $500 \mathrm{~m}$ coverage rate of bus stops in the network centre area decreased from $78.14 \%$ to $77.22 \%$ and $90.15 \%$ to $89.62 \%$, respectively. It can be seen that node deletion has no significant impact on the service level of the PTN. Therefore, the procedure for deleting a node is effective, and the node deletion has little impact on the subsequent TRP.

To verify the applicability of the proposed simplification method, two methods were used to simplify the PTN. The first one is cluster-growing renormalisation $(\mathrm{CG})$, which incrementally grows the super-nodes from seed nodes within a distance no larger than $c$ (in one super-node the distance between two nodes is at most $2 \cdot c+1$ steps, in this study, $c=1$ ) [18]. The second one is merging nodes based on community detection, where super-nodes are identified by communities revealed by balanced propagation (BP) [17]. The number of nodes in the network simplified by CG and BP methods are both less than $40 \%$ of the original, and all of them are super-nodes. Although the simplification effect of is better, if the TRP is carried out on the simplified network, the planning result will be abstract. Statistical analysis of the change in local network attributes of the simplified network obtained by different methods are shown in Figure 5. The results show that in comparison with $\mathrm{CG}$ and BP the proposed simplification method can effectively reduce the number of network nodes and edges, while retaining the local attributes of the network to a great extent.

\subsection{Determining the transfer coefficient}

To determine the appropriate parameters combination, five groups of transfer coefficients as shown in Table 2 were tested. The results showed that the five different PTNs contained 105, 101, 99, 98, and 94 routes, respectively. The comparison results of

Table 2 - The value of transfer coefficient in different groups

\begin{tabular}{|c|c|c|c|c|c|c||}
\hline Group number & $w_{1}^{\text {skeleton }}$ & $w_{2}^{\text {skeleton }}$ & $w_{1}^{\text {trunk }}$ & $w_{2}^{\text {trunk }}$ & $w_{1}^{\text {coverage }}$ & $w_{2}^{\text {coverage }}$ \\
\hline \hline 1 & 0.2 & 0 & 0.4 & 0 & 0.5 & 0.2 \\
\hline 2 & 0.4 & 0 & 0.8 & 0 & 1.0 & 0.4 \\
\hline 3 & 0.6 & 0 & 1.2 & 0 & 1.5 & 0.6 \\
\hline 4 & 0.8 & 0 & 1.6 & 0 & 2.0 & 0.8 \\
\hline 5 & 1.0 & 0 & 2.0 & 0 & 2.5 & 1.0 \\
\hline
\end{tabular}




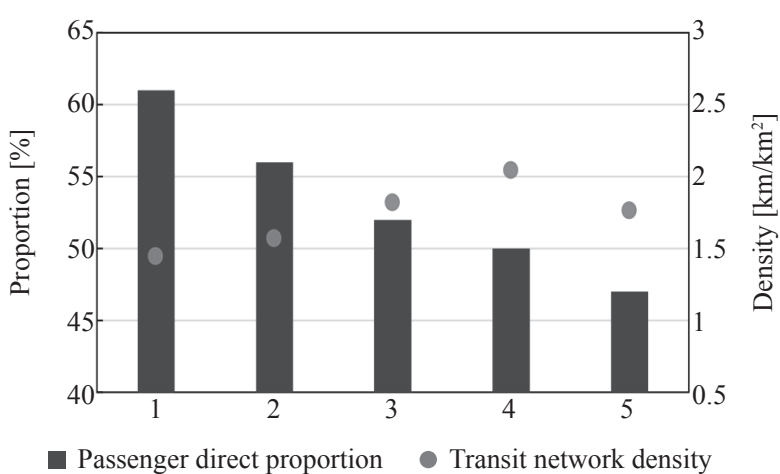

Figure 6-Passenger direct proportion and transit network density

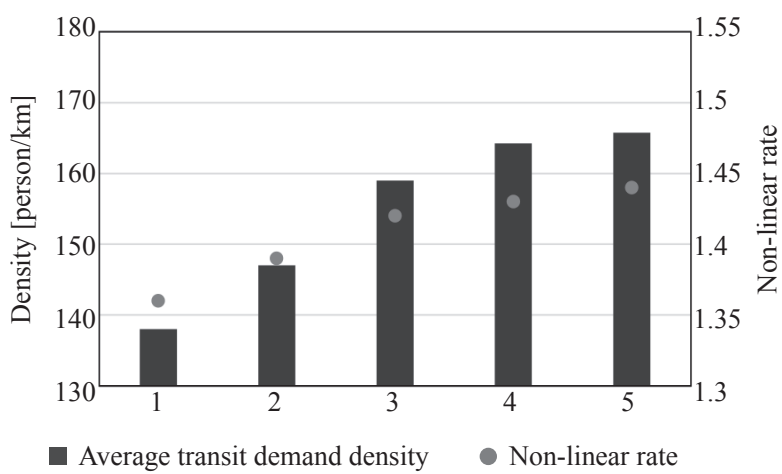

Figure 7 - Average transit demand density and non-linear rate

the passenger direct proportion, transit network density, non-linear rate, and average transit demand density of each network are shown in Figures 6 and 7.

Compared with other PTNs, in the first PTN the passenger direct proportion is the highest and the non-linear rate is the smallest. However, the network density and average transit demand density of the first PTN are the smallest. Therefore, the first PTN has the lowest service level. As the transfer parameter value increased, the passenger direct proportion decreased, and the network density increased continuously. The fourth PTN had the highest density, and the passenger direct proportion was $50 \%$, which meets the travel demands of most passengers in the network. The increase in transfer parameters leads to an increase in the non-linear rate and average transit demand density, which shows that the routes are more meandering and can meet higher passenger travel demands. The fourth and the fifth PTNs have the highest non-linear rate and highest average transit demand density of 1.44 and $1.45,164.24$, and 165.76, respectively. Therefore, group 4 was adopted in the TRP.

\subsection{Results and analysis}

With the best combination, there are 10 bus skeleton lines, 26 bus trunk lines, and 62 bus coverage lines in the network as shown in Figure 8. The bus skeleton lines mainly pass through commercial and residential areas with large passenger flow, forming an overall structure of "five horizontal and five vertical". The bus trunk lines, with transfer hubs as the supporting point, form the main skeleton of the PTN. The bus coverage lines meander into the community to meet the daily travel and commuting demand of residents.

To test the validation of the proposed TRP, methods for minimising the travel time method and maximising direct transit demand are introduced for comparison and solved by ACO. Table 3 lists the non-linear rate, passenger direct proportion, transit network density, multiple-line coefficient, and transfer coefficient of each planned PTN. In Table 3, the non-linear rate, transit network density, and transfer coefficient of the PTN planned by the proposed method are higher than others. In other words, the method pays more attention to the transfers, which leads to a network with a passenger direct proportion lower than that of other networks. Because the incremental allocation method is used to allocate the transit passenger, the number of bus lines on the road section is effectively controlled, hence, the multiple-line coefficient is the smallest.

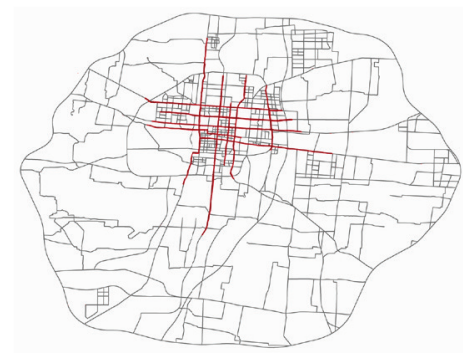

a) Bus skeleton line network

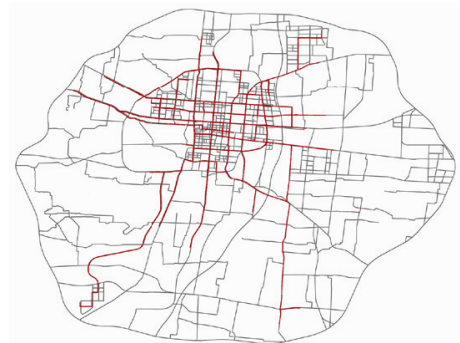

b) Bus trunk line networ

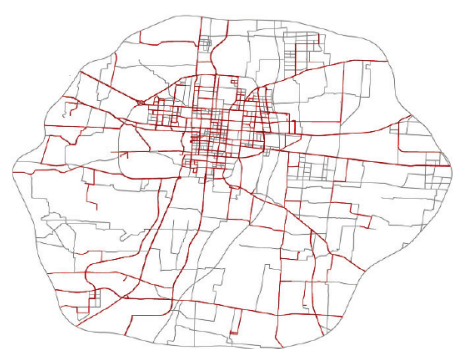

c) Bus coverage line network

Figure 8-Transit network with best transfer coefficients 
Pang M, Wang X, Ma L. Transit Route Planning for Megacities Based on Demand Density of Complex Networks

Table 3 - Comparison of transit network parameters

\begin{tabular}{|c|c|c|c|c|c|c|}
\hline \multirow{2}{*}{ Method } & \multirow{2}{*}{$\begin{array}{l}\text { Non-liner } \\
\text { rate }\end{array}$} & \multirow{2}{*}{$\begin{array}{c}\text { Passenger } \\
\text { direct } \\
\text { proportion }[\%]\end{array}$} & \multicolumn{2}{|c|}{$\begin{array}{c}\text { Transit network } \\
\text { density }\left[\mathrm{km} / \mathrm{km}^{2}\right]\end{array}$} & \multirow{2}{*}{$\begin{array}{l}\text { Line repetition } \\
\text { coefficient }\end{array}$} & \multirow{2}{*}{$\begin{array}{l}\text { Transfer } \\
\text { coefficient }\end{array}$} \\
\hline & & & Border & Centre & & \\
\hline Proposed method & 1.444 & 43.59 & 1.93 & 3.24 & 1.98 & 1.39 \\
\hline Minimise travel time & 1.435 & 45.36 & 1.87 & 3.17 & 2.02 & 1.31 \\
\hline Maximise direct transit demand & 1.441 & 47.13 & 1.91 & 3.22 & 2.07 & 1.36 \\
\hline
\end{tabular}

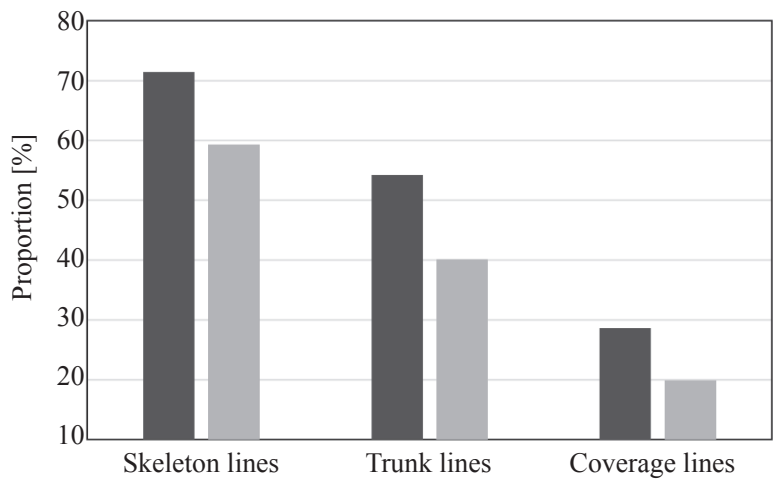

a) Proportion of bus lane

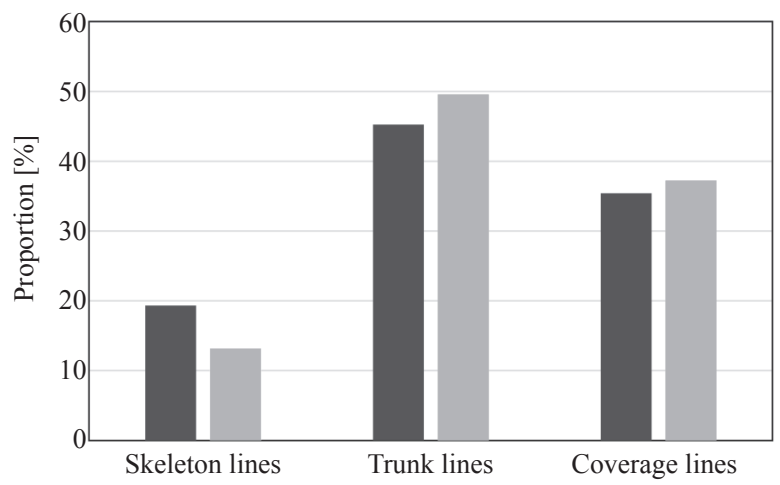

c) Proportion of transit demand

Planned network

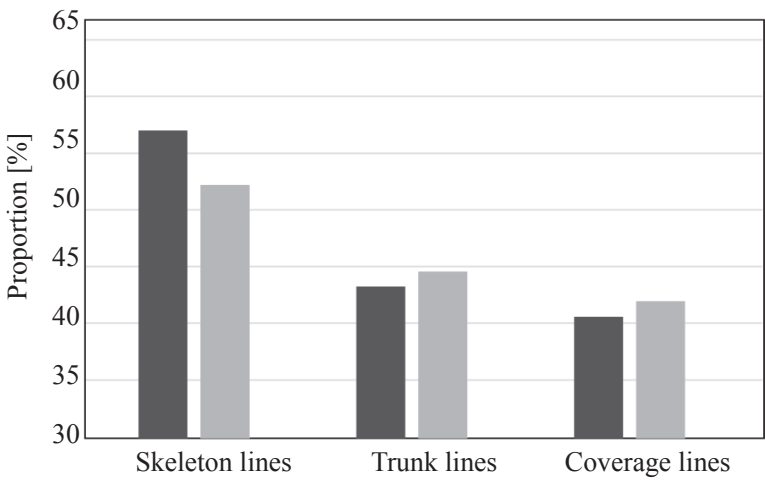

b) Proportion of passenger direct

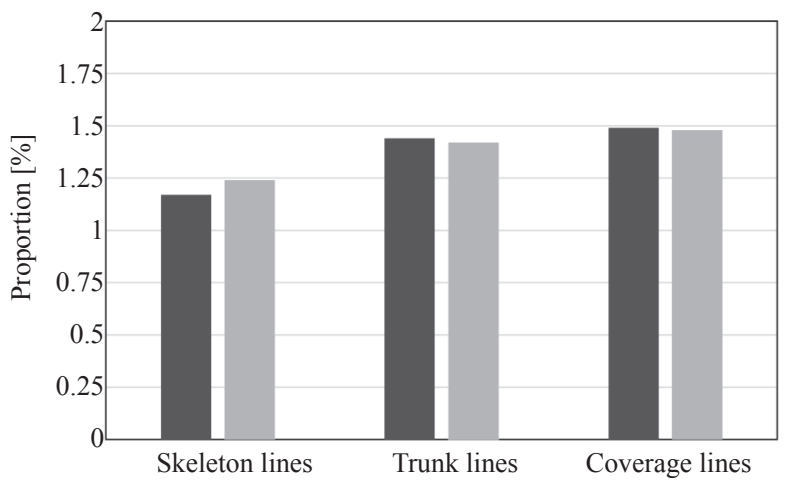

d) Non-linear rate

Existing network

Figure 9 - Comparison of bus lines at different layers between planned and existing network

Figure 9 shows the bus lane proportion, passenger direct proportion, transit demand proportion, and non-linear rate carried by three layers of bus lines in the existing and planned network. It is obvious that in each layer of the planned routes, the bus lane proportion gradually increased, while the passenger direct proportion are gradually decreased. The skeleton lines, despite being the least in number, still carried approximately $19.32 \%$ of the demand, which is $6.14 \%$ higher than that of the existing network. Moreover, the bus lane proportion and the passenger direct proportion improved, and the non-linear rate was reduced, which fully exploits the advantages of fast, direct, and large capacity. The bus trunk lines carried the most demands in both existing and planned network. Compared with the existing network, the direct passenger proportion was reduced and the non-linear rate increased, which is mainly attributed to the increased attention given to the transfers. In the planned network, the passenger direct proportion of three bus line layers increased and significantly changed between different layers, although there was little change in the existing network. This suggests that the route hierarchy in the planned network is clearer. More than half of the demands carried by bus coverage lines needs to be transferred. The non-linear rate obviously increased, showing that the bus line goes deep into the community, further expanding the coverage and accessibility of the PTN. Overall, the proposed 
Pang M, Wang X, Ma L. Transit Route Planning for Megacities Based on Demand Density of Complex Networks

TRP method can effectively increase the service area of the PTN, optimise the network structure, and improve the convenience of operator management.

\section{CONCLUSION}

Using the transit demand density of the edge of a network, a network simplification method for PTN for megacities was developed. A TRP model aimed to maximise the transit demand density of the network was established, which considers the transfers, especially the transfer demand of passengers at different stops. The steps in the optimisation solution of the TRP problem were established based on ACO. Under the objective function with different transfer coefficients and constraint conditions, the skeleton routes, trunk routes, and coverage routes were planned layer by layer to form the PTN. The public transport system data of Handan City in China were used for the test. The results show that the simplified PTN retained the local attributes of the original network to a great extent. The TRP method can make the route hierarchy of PTNs clearer to further improve the network service level. However, there are some issues that need to be resolved. This study is devoted to simplifying the PTNs and optimising the network structure, but does not take into account the price of passengers routing selection. Moreover, the incremental assignment method was used as an approximate equilibrium assignment method, which may lead to a deviation between the planning results and the actual situation. Therefore, it is necessary to develop an appropriate equilibrium assignment method in further studies.

\section{ACKNOWLEDGMENTS}

The work described in this paper was supported by the National Natural Science Foundation of China (50478088), the Natural Science Foundation of Hebei Province in China (E2015202266), and Transportation Science and Technology Development Plan Project of Tianjin (2018R36). The authors would like to gratefully acknowledge the editor's comments and the referees of the paper who helped to clarify and improve the presentation.

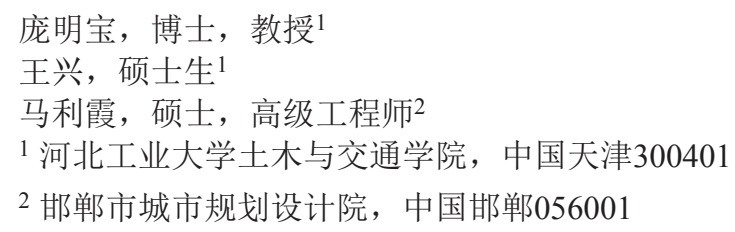

基于复杂网络需求密度的大城市公交线路规划

\section{摘要:}

本研究旨在探讨大城市公交网络简化和线路规划 问题。根据复杂网络需求密度, 制定了公交网络中 心区域的节点删除规则和网络边界区域的节点合并 规则。建立了公交线路规划模型, 该模型考虑了直 达乘客、同站换乘乘客和不同站点换乘乘客的传输 需求, 以最大化网络传输需求密度为目标。提出了 一种基于蚁群算法的线路规划方法。以邯郸市为例 验证了该方法的有效性。结果表明, 简化方法在有 效减小网络规模的同时很大程度上保留了其局部属 性。分层规划使得网络的层次结构更加清晰, 并有 效地提高城市公交网络的运行效率和服务水平。

\section{关键词:}

城市公交; 网络简化; 公交线路规划;

复杂网络需求密度; 蚁群优化算法

\section{REFERENCES}

[1] $\mathrm{He} \mathrm{H}$, et al. Providing public transport priority in the perimeter of urban networks: A bimodal strategy. Transportation Research Part C: Emerging Technologies. 2019;107: 171-192. doi: 10.1016/j.trc. 2019.08.004.

[2] Ceder A, Wilson N. Bus network design. Transportation Research Part B: Methodological. 1986;20(4): 331-344. doi: 10.1016/ 0191-2615(86)90047.

[3] Zhou K, et al. Multi objective optimization method of public transit networks based on travel behavior. Journal of Highway \& Transportation Research \& Development. 2015;9(4): 71-77. doi: 10.1016/ JHTRCQ.0000473.

[4] Carrese S, Gori S. An urban bus network design procedure. Transportation Planning. 2002;1(6): 177-195. doi: 10.1007/ 0-3-6-48220-7_11.

[5] Oloveora Arbex R, Barbieri da Cunha C. Efficient transit network design and frequencies setting multi-objective optimization by alternating objective genetic algorithm. Transportation Research Part B: Methodological. 2015;81: 355-376. doi: 10.1016/ j.trb.2015.06.014.

[6] Lai D, Caliskan Demirag O, Leung J. A tabu search heuristic for the heterogeneous vehicle routing problem on a multigraph. Transportation Research Part E: Logistics and Transportation Review. 2016;86: 32-52. doi: 10.1016/ j.tre.2015.12.001.

[7] Dong X, Dong W, Cai Y. Ant colony optimisation for coloured travelling salesman problem by multi-task learning. IET Intelligent Transport Systems. 2018;12(8): 774-782. doi: 10.1016/ iet-its.2016.0282.

[8] Zhang Z, et al. Spatial-temporal traffic flow pattern identification and anomaly detection with dictionary-based compression theory in a large-scale urban network. Transportation Research Part C: Emerging Technologies. 2016;7(10): 284-302. doi: 10.1016/j. trc.2016.08.0 06 .

[9] Li M, et al. Identifying essential proteins based on sub-network partition and prioritization by integrating subcellular localization information. Journal of 
Pang M, Wang X, Ma L. Transit Route Planning for Megacities Based on Demand Density of Complex Networks

Theoretical Biology. 2018;447(3): 65-73. doi: 10.1016/ j. jtbi.2018.03.029.

[10] Zhang W, Xu W. Simulation-based robust optimization for the schedule of single-direction bus transit route: The design of experiment. Transportation Research Part E: Logs and Transportation Review. 2017;106(10): 203-230. doi: 10.1016/ j.tre.2017.08.001.

[11] Yan G, et al. Efficient routing on complex networks. Physical Review E: Statistical Nonlinear \& Soft Matter Physics. 2005;73(2): 1-5. doi: 10.1103/ PhysRevE.73.046108.

[12] Zhang L, et al. An auxiliary optimization method for complex public transit route network based on link prediction. Modern Physics Letters B. 2018;32(5): 1850066. doi: 10.1142/ S0217984918500665.

[13] Jia G, Ma R, Hu Z. Urban transit network properties evaluation and optimization based on complex network theory. Sustainability. 2019;11(7): 1-16. doi: 10.3390/ su11072007.

[14] Clauset A, Newman M, Moore C. Finding community structure in very large networks. Physical Review E. 2004;70(2): 066111. doi: 10.1103/ PhysRevE.70.06611.

[15] Rolls D, Robins G. Minimum distance estimators of population size from snowball samples using conditional estimation and scaling of exponential random graph models. Computational Stats \& Data Analysis. 2017;116(12): 32-48. doi: 10.1016/ j.csda.2017.07.004.

[16] Boobalan P, Lopez D. Graph clustering using k-Neighbourhood Attribute Structural similarity. Applied Soft Computing. 2016;47(6): 216-223. doi: 10.1016/ j.asoc.2016.05.028.

[17] Blagus N, Šubelj L, Bajec M. Assessing the effectiveness of real-world network simplification. Physica A: Statal Mechanics and its Applications. 2014;413(7): 134-146. doi: 10.1016/ j.physa.2014.06.065.

[18] Gallos L, Song C, Makse H. A review of fractality and self-similarity in complex networks. Physica A: Statistical Mechanics and its Application. 2016;386 (2): 686691. doi: 10.1016/ j.physa.2007.07.069.
[19] Šubelj L, Bajec M. Robust network community detection using balanced propagation. European Physical Journal B. 2011;81(3): 353-362. doi: 10.1140/epjb/ e2011-10979-2.

[20] Lownes N, Machemehl R. Exact and heuristic methods for public transit circulator design. Transportation Research Part B: Methodological. 2010;44(2): 309-318. doi: 10.1016/j.trb.200 9.07.010.

[21] Yu B, et al. Transit route network design-maximizing direct and transfer demand density. Transportation Research Part C: Emerging Technologies. 2012;22(12): 5875. doi: 10.1016/j.trc.2011.12.003.

[22] Ibarra-Rojas OJ, et al. Planning, operation, and control of bus transport systems: A literature review. Transportation Research Part B: Methodological. 2015;77(7): 3875. doi: 10.1016/j.trb.2015. 03.002.

[23] Wang D, Nayan A, Szeto WY. Optimal bus service design with limited stop services in a travel corridor. Transportation Research Part E: Logs and Transportation Review. 2018;111(3): 70-86. doi: 10.1016/ j.tre.2018.01.007.

[24] Li J, Zheng P, Zhang W. Identifying the spatial distribution of public transportation trips by node and community characteristics. Transportation Planning and Technology. 2020;43(3): 1-16. doi: 10.1080/03081060.2020.1735776.

[25] Manser P, et al. Designing a large-scale public transport network using agent-based microsimulation. Transportation Research Part A: Policy and Practice. 2020;137(7): 1-15. doi: 10.1016/j.tra.2020.04.011.

[26] Yang Z, Yu B, Cheng C. A parallel ant colony algorithm for bus network optimization. Computer-aided Civil and Infrastructure Engineering. 2007;22(1): 44-55. doi: 10.1111/j.146 7-8667.2006.00469.x.

[27] Agrawal J, Mathew T. Transit route network design using parallel genetic algorithm. Journal of Computing in Civil Engineering. 2004;18(3): 248-256. doi: 10.1061/ (ASCE)0887-3801(2004)18:3(248).

[28] Dorigo M, Maniezzo V, Colorni A. Ant system: Optimization by a colony of cooperating agents. IEEE Transactions on Systems Man and Cybernetics. 1996;26(1): 29-41. doi: 10.1109/3477.484436. 